\title{
Attitudes of Physicians in Jordan Towards Non-Disclosure of Health Information
}

\author{
"Saif M. Borgan, ${ }^{1}$ Justin Z. Amarin, ${ }^{2}$ Areej K. Othman, ${ }^{3}$ Haya H. Suradi, ${ }^{2}$ Yasmeen Z. Qwaider ${ }^{2}$
}

\begin{abstract}
Objectives: This report aims to investigate the attitudes of physicians in Jordan towards non-disclosure of health information among physicians, with a focus on those who are 'always truthful' and those who are not. Methods: The report is based on the second subset of data from a cross-sectional study-conducted between January and August 2016 - of the truth disclosure practices among and attitudes of physicians in Jordan. The sample consisted of 240 physicians selected from four major hospitals by stratified random sampling and invited to complete a selfadministered questionnaire regarding truth disclosure attitudes. The attitudes of physicians who were 'always truthful' were compared with those who were not. Results: A total of 164 physicians (response rate: 68\%) completed the questionnaire. Of these, 17 (10\%) were 'always truthful', while the remaining 144 (90\%) were not. Physicians who were 'always truthful' were more likely to indicate that non-disclosure is 'unethical' ( $77 \%$ versus $39 \% ; P=0.009$ ). Moreover, physicians who were 'always truthful' were more likely to disagree that non-disclosure is beneficial for the physical and psychological health of patients ( $82 \%$ versus $55 \% ; P=0.03$ ). Most of the surveyed physicians agreed that all patients have the right to know their diagnosis, most patients prefer to know their diagnosis and the introduction of legislation to enforce disclosure would positively affect medical practice in Jordan. Conclusion: The differential attitudes of physicians who were 'always truthful' and those who were 'not always truthful' suggests a rationale behind independent non-disclosure; namely, that non-disclosure is ethically justifiable and beneficial for the physical and psychological health of patients.
\end{abstract}

Keywords: Truth Disclosure; Physicians; Attitude; Middle East; Cross-Sectional Studies; Jordan.

\section{AdVANCES IN KNOWLEDGE}

Physicians who were 'not always truthful' were more likely to agree that non-disclosure is beneficial for the physical and psychological health of patients, which suggests that therapeutic privilege is one possible ethical rationale for non-disclosure.

Of the physicians who were 'not always truthful', 39\% indicated that non-disclosure is 'unethical', which suggests that these physicians may feel pressured to comply with sociocultural norms that they do not necessarily agree with.

\section{Application to Patient Care}

Physicians who were 'always truthful' and those who were 'not always truthful' generally believed that the introduction of legislation to enforce disclosure would positively affect medical practice (and therefore, patient care) in Jordan.

$\mathrm{N}$ ON-DISCLOSURE OF HEALTH INFORMATION was widely prevalent in $20^{\text {th }}$-century Western medicine. ${ }^{1,2}$ In 1961, Oken reported that 90\% of a sample of physicians in the United States indicated a preference for not telling cancer patients they have cancer. ${ }^{2}$ Non-disclosure has since dramatically decreased in the United States and is unjustified by contemporary ethics. ${ }^{3,4}$ In many courts, nondisclosure is considered negligent and illegal and 'therapeutic privilege' is not a valid defence because discretion cannot take precedence over informed consent. ${ }^{4}$ However, non-disclosure remains prevalent in more conservative countries including Japan, China, Pakistan and many countries in the Middle East. ${ }^{5-8}$

In these countries, multiple factors motivate truth non-disclosure; however, sociocultural factors, such as strong family influence, appear to be the main drivers. ${ }^{9,10}$ In the context of cancer, for example, families often request non-disclosure because of the associated stigma, misconceptions regarding prognosis and their perceptions of the negative effect of disclosure on patient well-being. ${ }^{7}$ Non-disclosure practices are further propagated by the lack of transparent legislation to protect patient autonomy.

From the perspective of physicians, disclosure may theoretically conflict with other ethical principles, such as beneficence and non-maleficence. For instance, some physicians believe that informing patients with a terminal illness of their prognosis results in 'unnecessary' psychological suffering and is medically contraindicated. ${ }^{1,11}$ Non-disclosure motivated by this reason is termed 'therapeutic privilege."

Similar to other countries in the Middle East, Jordan's culture is heavily influenced by family-centred values. The current researchers previously conducted a cross-sectional study to investigate truth disclosure practices of physicians in Jordan. ${ }^{10}$ In 2018, the researchers reported that $23 \%$ of physicians do not 
usually disclose the truth to a patient with a serious illness and are commonly motivated by a direct request from the patient's family. However, $15 \%$ of physicians who do not usually disclose the truth do so independently-with no impetus from the patient's family-and current evidence does not explain the rationale behind independent non-disclosure in Jordan. ${ }^{10}$ This study aimed to investigate the attitudes of physicians in Jordan towards non-disclosure, with a secondary focus on the differences in attitude between those who are 'always truthful' and those who are not.

\section{Methods}

The current investigation is based on the second subset of data from a cross-sectional study of truth disclosure practices and attitudes of physicians in Jordan, which was conducted between January and August 2016. ${ }^{10}$ The researchers' previous report explored the truth disclosure practices of physicians in Jordan. In the current study, the attitudes of physicians who always disclose the truth are compared with those who do not.

The physicians employed by two of the largest hospitals from each of the public and private sectors in Amman, the capital of Jordan, were surveyed. The total bed capacity of the four hospitals is $1,524 .{ }^{10}$

The lists of physicians employed by each hospital were obtained and used to select the preliminary sample by stratified random sampling. Paediatricians and psychiatrists were excluded because they deal with unique and specific truth disclosure considerations. Sixty participants from each participating hospital were randomly selected using an online random number generator based on atmospheric noise for a total preliminary sample of 240 participants. The participants were approached during office hours, introduced to the study and invited to participate by completing a self-administered questionnaire.

A questionnaire developed by Hamadeh and Adib was modified, following permission, to accommodate the sample. ${ }^{12}$ The questionnaire includes closed-ended and Likert-type questions regarding attitudes towards non-disclosure of health information. The language of complex and ambiguous questions was respectively simplified and clarified, as identified by a pilot sample of 15 physicians employed by a small private hospital in Amman. Two independent experts were also asked to review the face and content validity of the questionnaire.

Statistical Package for the Social Sciences (SPSS), Version 23.0 (IBM Corp., Armonk, New York, USA) was used to perform data analysis. First, descriptive statistics were computed. Second, physicians were stratified according to their disclosure policy: 'always truthful' versus 'not always truthful'. Physicians whose usual disclosure policy was to 'informed' and who reported never making exceptions to their policy were considered 'always truthful'. Physicians who reported a usual 'do not inform' policy or those who reported a 'informed' policy but reported making exceptions of any frequency were considered 'not always truthful.' Finally, the association between questions concerning disclosure policy and truth disclosure attitude was investigated using Pearson's $X^{2}$ test and values of $P$ $<0.05$ were interpreted as statistically significant. Throughout the report, numerical data are presented according to the recommendations of Cole..$^{13}$

The ethics committees of all participating hospitals approved the study protocol and waived the requirement for written consent. Verbal consent from each participant was obtained.

\section{Results}

Out of 240 invitees, 164 (response rate: 68\%) completed the questionnaire. The characteristics of the sample have been described previously. ${ }^{10}$ It was found that 126 physicians (77\%) were non-specialists and 38 (23\%) were specialists; 113 (69\%) were men and 51 (31\%) were women; and 95 (58\%) worked in the private sector while 69 (42\%) worked in the public sector [Table 1]. The usual disclosure policy of 127 physicians (77\%) was to inform, compared with 37 (23\%) whose usual disclosure policy was not to inform. According to their reported disclosure policy, 17 physicians (10\%) were 'always truthful', while the remaining 147 (90\%) were not [Table 2].

In total, 19 physicians (12\%) indicated that nondisclosure is ethical, 70 (43\%) indicated that nondisclosure is unethical and 75 (46\%) indicated that it depends on the situation. Physicians who were 'always truthful' were more likely to indicate that non-disclosure is unethical (77\% versus 39\%; $\chi^{2}(2)=$ 9.3, $P=0.009$ ). Notably, none of the physicians who were 'always truthful' indicated that non-disclosure is ethical. In addition, 69 physicians (42\%) agreed that non-disclosure is beneficial for the physical and psychological health of patients, while 95 (58\%) disagreed [Table 2]. Physicians who were 'always truthful' were more likely to disagree (82\% versus 55\%; $\left.X^{2}(1)=4.7, P=0.03\right)$.

Among the physicians belonging to the not always truthful' group, when asked if they agreed that 'all patients have the right to know their diagnosis', 94 (57\%) strongly agreed, 61 (37\%) agreed, seven (4\%) were neutral and two (1\%) disagreed. These physicians were also asked if they agreed that 'most patients prefer to know their diagnosis' and 59 (36\%) strongly agreed, 75 
Table 1: Characteristics of the included physicians from four hospitals in Amman, Jordan ( $\mathrm{N}=164)$

\begin{tabular}{lc} 
Characteristic & $\mathbf{n}(\%)$ \\
Age group & \\
20-29 years & $97(59)$ \\
$30-39$ years & $41(25)$ \\
$40+$ years & $26(16)$ \\
Gender & \\
Male & $113(69)$ \\
Female & $51(31)$ \\
Employment sector & \\
Public & $69(42)$ \\
Private & $95(58)$ \\
Nationality & \\
Jordanian & $144(88)$ \\
Other & $20(12)$ \\
Marital status & \\
Single & $85(52)$ \\
Engaged & $8(5)$ \\
Married & $69(42)$ \\
Other & $22(1)$ \\
Level of specialisation & \\
General practitioner & $31(19)$ \\
Resident & $95(58)$ \\
Specialist & $38(23)$ \\
Physician experience & \\
1-5 years & years \\
\hline
\end{tabular}

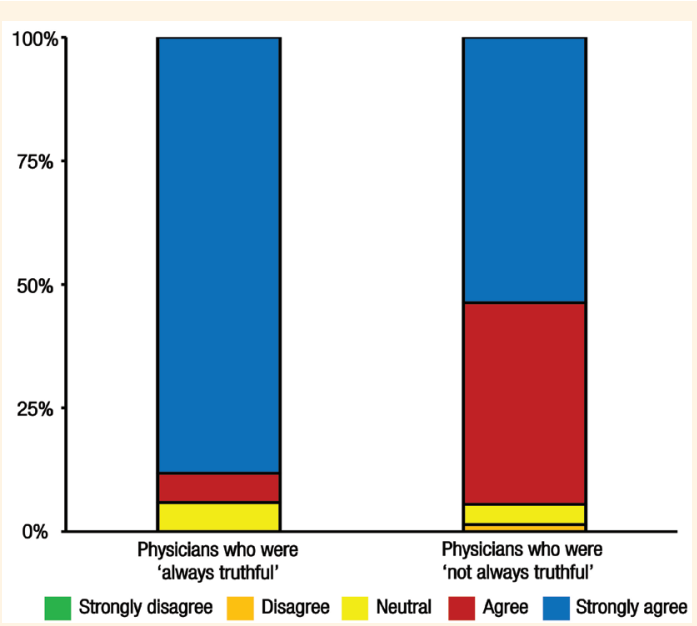

Figure 1: Ratings of 164 physicians from Amman, Jordan, regarding their perception of a patient's right to know their diagnosis stratified by disclosure policy using a Likert-type scale.
Table 2: Attitudes towards non-disclosure of medical information among included physicians from four hospitals in Amman, Jordan

$\begin{aligned} & \text { Attitude } \\ & \text { Policy on disclosing the diagnosis of 'serious illness' directly } \\ & \text { to a competent adult patient }\end{aligned}$
$\begin{aligned} & \text { Inform } \\ & \text { Do not inform }\end{aligned}$
$\begin{aligned} & \text { Consistency of truthfulness } \\ & \text { Always truthful }\end{aligned}$
$\begin{aligned} & \text { Not always truthful } \\ & \text { Perceived ethics of non-disclosure* }\end{aligned}$
$\begin{aligned} & \text { Ethical } \\ & \text { Unethical }\end{aligned}$
$\begin{aligned} & \text { Depends on the situation } \\ & \begin{array}{l}\text { Perceived physical and psychological benefit of non- } \\ \text { disclosure to patients* }\end{array}\end{aligned}$
$\begin{aligned} & \text { Beneficial } \\ & \text { Not beneficial }\end{aligned}$
$\begin{aligned} & \text { "Statistically significant difference in this attitude between physicians who } \\ & \text { are always truthful' and those who are not. }\end{aligned}$

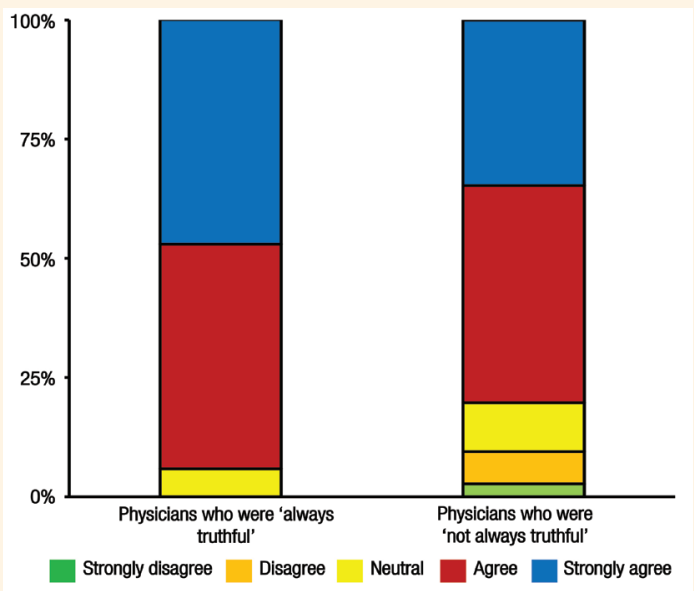

Figure 2: Ratings of 164 physicians from Amman, Jordan, regarding their perception of a patient's preference to know their diagnosis stratified by disclosure policy using a Likert-type scale.

(46\%) agreed, 16 (10\%) were neutral, 10 (6\%) disagreed and four (2\%) strongly disagreed. The distribution of responses for physicians who were 'always truthful' and those who were 'not always truthful' are presented in Figures 1 and 2.

If they were to fall seriously ill, 140 physicians (85\%) indicated that they would want to be informed of their diagnosis directly, while 19 (12\%) indicated that they would want their family to make the decision to inform them or not and five (3\%) indicated that they would not want to be informed at all. The distribution 
of responses in favour of direct disclosure was similar for physicians who were 'always truthful' and those who were 'not always truthful' (94\% and 84\%, respectively; $\left.X^{2}(1)=1.2, P=0.3\right)$.

Finally, 151 physicians (92\%) believed that the introduction of legislation to enforce disclosure would positively affect medical practice in Jordan, while 12 (8\%) did not. Once again, the distribution of positive responses was similar for physicians who were 'always truthful' and those who were 'not always truthful' (94\% versus 92\%; $X^{2}(1)=0.1, P=0.7$ ). Compared with physicians working in the private sector, those working in the public sector were more likely to believe that the introduction of legislation to enforce disclosure would positively affect medical practice in Jordan (87\% versus 99\%; $\left.\chi^{2}(1)=6.8, P=0.009\right)$.

\section{Discussion}

In this study, the attitudes of physicians in Jordan towards non-disclosure of health information are explored with a secondary focus on the difference in attitudes between those who were classified as 'always truthful' and those who were not. It was found that physicians who were 'always truthful'- the minoritywere more likely to indicate that non-disclosure is unethical and unbeneficial for the physical and psychological health of patients. Most of the surveyed physicians (including those who were 'not always truthful') indicated that all patients have the right to know their diagnosis, most patients prefer to know their diagnosis and the introduction of legislation to enforce disclosure would positively affect medical practice in Jordan.

In Jordan, non-disclosure of health information is prevalent; the researchers previously reported that the usual policy of $23 \%$ of a sample of physicians was not to disclose. ${ }^{10}$ Sociocultural factors are often the drivers of non-disclosure. ${ }^{9,10}$ However, the attitudes of physicians may also be influential. For example, the researchers also showed that $15 \%$ of physicians who do not usually disclose the truth do so independently and that truth disclosure practices vary widely from hospital to hospital and even within the same hospital. ${ }^{10}$

In this study, it was found that none of the physicians who were 'always truthful' indicated that non-disclosure is ethical, which reflects a hard-line, black-and-white stance on truth disclosure. In the grey area, many people in the Middle East would not want the truth disclosed to them. ${ }^{8}$ Indeed, in a Lebanese study of 498 participants, including patients with cancer, patients with no cancer and healthy people, $42 \%$ indicated a preference for non-disclosure. ${ }^{14}$ However, recent evidence suggests that the attitudes of both physicians and the public are shifting in favour of personal autonomy. ${ }^{8,15}$ In support, the researchers of the current study also posed a reverse scenario and found that $85 \%$ of physicians indicated that they would want to be informed of their diagnosis directly if they were to fall seriously ill.

It was also found that most of the physicians surveyed as part of the present investigation, who were 'not always truthful', indicated that whether non-disclosure is ethical 'depends on the situation.' According to previous studies, a physician is less likely to disclose sensitive health information if the patient has a poor prognosis, such as end-stage cancer or limited life expectancy or if the physician is wary of characteristics that signal a limited ability to cope with the truth, such as advanced age or preference for nondisclosure. ${ }^{12,16,17}$ In these situations, physicians may invoke therapeutic privilege as the ethical rationale for non-disclosure; disclosure may be considered cruel and likely debilitative to patient well-being. Indeed, therapeutic privilege may inform the attitudes of both physicians and the public towards non-disclosure. ${ }^{8,16}$ In support, this study found that physicians who were 'not always truthful' were more likely to agree that non-disclosure is beneficial for the physical and psychological health of patients.

It was also found that some of the physicians who were 'not always truthful' indicated that nondisclosure is 'unethical'. These physicians may feel pressured to comply with sociocultural norms that they do not necessarily agree with. Based on the researchers' own observations, the repercussions of disclosing the diagnosis of a serious illness against the family's wishes are grave and the physician-familypatient relationship may break down as a result. In Eastern cultures, physicians often make medical decisions with input from the patient's family. ${ }^{18}$ Hence, they may be compelled to withhold information from the patient for the sake of maintaining the relationship with the family so the patient can continue to receive the necessary treatment.

It is not clear whether there is a culturally specific and realistic approach to truth disclosure in the Middle East, a deficiency that must be addressed in future studies. While further data are needed to develop an evidence-based approach, culturally specific guidelines based on experience are necessary to support patient autonomy and alleviate the fears and misconceptions of physicians regarding truth disclosure. This study shows that physicians who were 'always truthful' and those who were 'not always truthful' generally believe that the introduction of legislation to enforce disclosure would positively affect medical practice in Jordan. This finding has also been shown in a parallel 
study of physicians in Bahrain. ${ }^{19}$ It is expected that the guidelines will have a bigger impact on the public sector, as supported by the attitudes of physicians, because the private sector in Jordan operates under limited governmental and public policy oversight. However, the overwhelming majority of physicians within both sectors believed that introducing truth disclosure legislation would have a positive impact.

The main limitations of the current study are potential non-response bias and selection bias. For example, the attitudes of physicians who chose not to participate may be considerably different from those who did. In addition, the four selected hospitals are set in urban areas and the attitudes of physicians in rural areas may also be vastly different. Finally, the small number of physicians who were 'always truthful'though telling-limits the validity of the comparative analysis.

\section{Conclusion}

The differential attitudes of physicians who were 'always truthful' and those who were 'not always truthful' suggests a rationale behind independent non-disclosure, namely that non-disclosure is ethical and beneficial for the physical and psychological health of patients. Regardless of disclosure policy, the introduction of legislation to enforce truth disclosure appears to be welcomed by most physicians.

\section{FUNDING}

This work was supported by a grant from The University of Jordan Deanship of Scientific Research.

\section{CONFLICT OF INTEREST}

The authors declare no conflict of interest.

\section{AUTHORS' CONTRIBUTION}

SMB conceived the study. SMB and AKO designed the study and modified the questionnaire. JZA, HHS and YZQ collected the data. JZA performed the data analysis. SMB and JZA wrote the manuscript. All authors reviewed and approved the final version of the manuscript.

\section{References}

1. Gillon R. Telling the truth and medical ethics. Br Med J (Clin Res Ed) 1985; 291:1556-7. https://doi.org/10.1136/bmj.291.65 08.1556
2. Oken D. What to tell cancer patients: A study of medical attitudes. JAMA 1961; 175:1120-8. https://doi.org/10.1001/jama.1961.03 040130004002

3. Fallowfield LJ, Jenkins VA, Beveridge HA. Truth may hurt but deceit hurts more: Communication in palliative care. Palliat Med 2002; 16:297-303. https://doi.org/10.1191/0269216302pm 575oa.

4. Edwin AK. Don't lie but don't tell the whole truth: The therapeutic privilege - Is it ever justified? Ghana Med J 2008; 42:156-61.

5. Uchitomi Y, Yamawaki S. Truth-telling practice in cancer care in Japan. Ann N Y Acad Sci 1997; 809:290-9. https://doi.org/10.11 11/j.1749-6632.1997.tb48092.x.

6. Li S, Chou JL. Communication with the cancer patient in China. Ann NY Acad Sci 1997; 809:243-8. https://doi.org/10.1111/j.17 49-6632.1997.tb48087.x.

7. Malik IA, Qureshi AF. Communication with cancer patients: Experiences in Pakistan. Ann N Y Acad Sci 1997; 809:300-8. https://doi.org/10.1111/j.1749-6632.1997.tb48093.x.

8. Bou Khalil R. Attitudes, beliefs and perceptions regarding truth disclosure of cancer-related information in the Middle East: A review. Palliat Support Care 2013; 11:69-78. https://doi.org/10.10 17/S1478951512000107.

9. Ni YH, Alræk T. What circumstances lead to non-disclosure of cancer-related information in China? A qualitative study. Support Care Cancer 2017; 25:811-6. https://doi.org/10.1007/ s00520-016-3464-z.

10. Borgan SM, Amarin JZ, Othman AK, Suradi HH, Qwaider YZ. Truth disclosure practices of physicians in Jordan. J Bioeth Inq 2018; 15:81-7. https://doi.org/10.1007/s11673-018-9837-x.

11. Richard C, Lajeunesse Y, Lussier MT. Therapeutic privilege: Between the ethics of lying and the practice of truth. J Med Ethics 2010; 36:353-7. https://doi.org/10.1136/jme.2009.033340.

12. Hamadeh GN, Adib SM. Cancer truth disclosure by Lebanese doctors. Soc Sci Med 1998; 47:1289-94. https://doi.org/10.10 16/s0277-9536(98)00203-2

13. Cole TJ. Too many digits: The presentation of numerical data. Arch Dis Child 2015; 100:608-9. https://doi.org/10.1136/arch dischild-2014-307149.

14. Adib SM, Hamadeh GN. Attitudes of the Lebanese public regarding disclosure of serious illness. J Med Ethics 1999; 25:399-403. https://doi.org/10.1136/jme.25.5.399.

15. Naji F, Hamadeh G, Hlais S, Adib S. Truth disclosure to cancer patients: Shifting attitudes and practices of Lebanese physicians. AJOB Empir Bioeth 2015; 6:41-9. https://doi.org/10.1080/2329 4515.2014 .996265

16. Harrison A, Al-Saadi AM, Al-Kaabi AS, Al-Kaabi MR, AlBedwawi SS, Al-Kaabi SO, et al. Should doctors inform terminally ill patients? The opinions of nationals and doctors in the United Arab Emirates. J Med Ethics 1997; 23:101-7. https:// doi.org/10.1136/jme.23.2.101.

17. Ozdogan M, Samur M, Bozcuk HS, Coban E, Artac M, Savas B, et al. "Do not tell": what factors affect relatives' attitudes to honest disclosure of diagnosis to cancer patients? Support Care Cancer 2004; 12:497-502. https://doi.org/10.1007/s00520-0040633-2.

18. Al-Bahri A, Al-Moundhri M, Al-Azri M. The role of patients' families in cancer treatment decision-making: Perspectives among Eastern and Western families. Sultan Qaboos Univ Med J 2017; 7:e383-5. https://doi.org/10.18295/squmj.2017.17.04.001.

19. Jassim, G, Alakri, A, Alsayegh, R, Misselbrook, D. Practice and attitudes of physicians regarding disclosure of information to patients with serious illness. Glob J Health Sci 2019; 11:33-40. https://doi.org/10.5539/gjhs.v11n5p33. 